\title{
ARTICLES
}

\section{Pourquoi les théories discursives peuvent avoir du mal à entrer en classe de langue(s) étrangère $(s)$}

\author{
Why discursive theories may have difficulty in entering \\ foreign language classes
}

\author{
Elżbieta Gajewska \\ Université Pédagogique de Cracovie \\ egajewska@up.krakow.pl
}

\begin{abstract}
The fact that verbal expression is subject to both language and social norms is indisputable. However, the advances of discursive theories are slow to be integrated into didactic practice in a systematic way. The article attempts to identify reasons underlying such a state of affairs. It is argued here that the overabundance of reference theories and the different scientific traditions lead to the fragmentation of the object of the study and create false terminological friends. The use of units of conceptualization and analysis of discourse, such as the genre or type of discourse, sometimes poses problems in didactic practice. Finally, discursive norms evolve continuously, often creating hybrid forms. One of the solutions to the above-mentioned problems, proposed in this article, is to make learners aware of discursive phenomena rather than try to provide ready-made solutions.
\end{abstract}

Keywords: Discourse, Genre, Genre Analysis, Discursive competence

Il y a déjà cinquante ans, Dell Hymes $(1966,1984)$ a constaté que les règles grammaticales ont besoin d'être complétées par des règles d'emploi. Afin d'être admis comme acceptable, un énoncé doit être approprié au contexte situationnel et socioculturel, Hymes propose donc de compléter la compétence linguistique de Noam Chomsky (1965) par des normes d'adéquation sociolinguistiques. La notion 
de compétence communicative a été bien vite reprise par la glottodidactique, où elle est devenue un concept de référence, et où elle a même donné nom à un courant méthodologique de portée internationale.

Actuellement, les liens entre le texte et son contexte situationnel sont analysés dans le cadre des «études de discours» (Maingueneau, 2014). En 2004, Zając (2004) voyait dans l'adoption d'une «approche discursive » une solution qui permettrait à la didactique des langues étrangères (désormais LE) le renouvellement de ses visées théoriques et ses démarches en classe. Cependant, l'approche communicative a été suivie par celle actionnelle, et la compétence discursive n'est qu'une des composantes de la compétence pragmatique du Cadre Européen Commun de Référence pour les Langues (désormais CECRL ; Conseil de l'Europe, 2001).

Tout en partageant la conviction que les perspectives discursives pourraient enrichir considérablement la recherche, nous voyons également des obstacles qui peuvent s'opposer à leur mise en pratique réelle dans l'enseignement. Ainsi, nous allons présenter certaines difficultés qui, à notre avis, peuvent gêner le transfert des acquis des études sur les discours dans le champ glottodidactique. Toute pratique de classe réfléchie devrait résulter d'un cadre conceptuel pertinent : pour cette raison, nous allons commencer par une réflexion sur les courants discursifs susceptibles de fournir un tel cadre aux enseignants de langues étrangères (désormais LE). Par la suite, nous allons examiner les concepts qui peuvent servir à des modélisations glottodidactiques, soit les «unités» de discours (Maingueneau, 2005). Finalement, nous allons nous pencher sur la nature même des genres du discours, et les conséquences qui en résultent pour leur didactisation éventuelle.

\section{LA PLURALITÉ DES APPORTS THÉORIQUES : RICHESSE OU EMBARRAS DE RICHESSE?}

Les problèmes avec le discursif commencent déjà au niveau du choix de l'appareil théorique de référence. Candlin (1993, p. IX) ${ }^{1}$ voit dans le discours un des gagnants sûrs d'un jeu dans lequel on chercherait les mots le plus souvent utilisés dans le milieu académique: toutefois, le problème se pose lorsqu' on voudrait déterminer sa signification exacte et les raisons de sa popularité. Quoiqu'on utilise l'appellation «analyse du discours » (désormais AD, cf. Charaudeau, Maingueneau, 2002), ce terme ne renvoie pas à une discipline. Les courants qui partagent une vision de la communication comprise comme une pratique sociale et se dédient

\footnotetext{
${ }^{1}$ One of the most diverting and harmless parlour-games one can play in any discipline is to calculate the current popularity and currency of particular terms - a kind of academic Trivial Pursuit. If one were to embark now on such an entertainment, a term like "discourse analysis" would have to be a sure-fire winner. The trouble would only come when the winners have to decide what it meant, and the reasons for its popularity (Candlin, 1993, p. IX).
} 
à l'analyse des énoncés en contexte de leur production sont légion. Boutet et Maingueneau (2005) énumèrent des approches qui se réclament directement de l'analyse du discours (École française althusérienne), conçues dans une perspective linguistique (grammaire du texte, théories de l'énonciation), qui oscillent entre anthropologie et sociolinguistique (ethnographie de la communication) ou sociologie et ethnométhodologie (analyse conversationnelle). Maingueneau (2005) pointe également qu'on a tendance à classer entre les approches discursives des disciplines ou courants (telles la sémiotique, stylistique, ethnographie de la communication, analyse critique du discours...), des composants obligés des interactions verbales (comme la proxémique, politesse, actes de langage...) ou finalement des conceptions du langage qui ne sont pas propres à un courant (telle la pragmatique).

\subsection{LES ENSEIGNANTS DE LANGUES ÉTRANGÈRES FACE À DES PERSPECTIVES MULTIPLES ET UN OBJET D'ÉTUDE MORCELÉ}

Le fait que, malgré leur convergence, les études discursives sont relativement indépendantes ne peut pas rester sans incidence sur leur adoption en classe de langue. En fait, une approche plurielle qui peut enrichir l'analyse des phénomènes linguistiques va se montrer problématique en pratique didactique du fait des connaissances préalables qu'elle nécessite, ceci aussi bien de la part de l'enseignant que des apprenants.

L'application des études discursives à l'enseignement des langues étrangères implique un passage obligatoire par la familiarisation des enseignants avec les avancées scientifiques respectives. Rien que les deux énumérations citées dans le passage examiné ci-dessus montrent l'ampleur de la tâche, car chaque courant examine la communication en adoptant un angle d'attaque particulier. Pour ne citer que deux exemples, l'analyse des conversations se focalise sur la gestion des tours ou le travail des faces, tandis que la linguistique textuelle étudie entre autres les schémas textuels et les procédés de cohérence et de cohésion. Des méthodologies distinctes entraînent le morcellement de l'objet d'analyse : la communication se scinde en écrite et orale, où les deux sphères nécessitent des outils d'analyse divers.

Ce morcellement ne contribue pas certes à rendre l'étude du discours plus aisée. Or, l'initiation des futurs professeurs de LE à l'analyse du discours n'est qu'un premier pas : il faudrait encore rendre l'appareil conceptuel choisi accessible au large public. La formation en sciences du langage des spécialistes de l'enseignement de celui-ci devrait forcément être accompagnée par des savoirs et des savoir-faire qui leur permettraient d'adapter ces connaissances ad usum Delphini, c'est-à-dire de les ajuster au développement cognitif des (souvent très) jeunes écoliers, ou bien de les accommoder aux attentes des professionnels dont l'intérêt pour la LE est souvent assez utilitaire et qui rechignent devant un métalangage qu'ils trouvent superflu. 


\subsection{L'HÉTÉROGÉNÉITÉ DES TRADITIONS SCIENTIFIQUES : UNE SOURCE POSSIBLE D'INCOMPRÉHENSION ENTRE LES ENSEIGNANTS DE LANGUES DIVERSES}

La profusion des courants de référence n'est pas le seul obstacle pour une adoption réelle du discursif dans l'enseignement des LE. Un autre problème de taille auquel on est bien vite confronté est l'hétérogénéité des traditions scientifiques et intellectuelles, dont la répartition est très souvent géographique ou langagière. Ainsi, il est possible de parler de «tendances françaises » ou «allemandes », même si, évidemment, toutes les recherches dans le domaine de l'analyse du discours menées dans ces pays (ou en ces langues) ne sont pas ancrées dans une seule méthodologie.

Les recherches en langue allemande adoptent souvent une perspective textologique (Czachur, Miller, 2002). L'exploration des paramètres qui relèvent de l'analyse du discours est effectuée dans le cadre de l'étude du texte (Textlinguistik, mais aussi Textanalyse, Textgrammatik ou Textwissenschaft). Quant au terme «discours»(Diskurs), il renvoie aux interactions orales, domaine de recherche abordé dans d'autres sphères langagières par le biais de l'analyse conversationnelle. Les recherches en langue anglaise rassemblées sous la bannière de la Discourse Analysis préfèrent l'entrée fonctionnelle ou sociolinguistique (Sanders, 1996). Elles ont très souvent des visées didactiques et contrastives : dans le monde anglo-saxon, les tentatives d'identifier l'organisation textuelle et les caractéristiques linguistiques de surface propres à des genres écrits ou oraux sont entreprises dans le but de les rendre accessibles à l'apprenant à des fins de compréhension et de production de textes ou d'interactions similaires. Tout comme dans la sphère de recherches de langue allemande, un important pan de recherches porte sur les discours spécialisés ou professionnels (Gajewska, Sowa, 2014, pp. 60-62). Quant à l' «École française d'analyse du discours », elle a choisi une perspective distincte. Sa particularité résidait dans le fait d'appliquer une approche analytique qui décomposait les totalités pour accéder au sens, tandis que l'approche intégrative communément adoptée en AD articule le discours «comme réseau d'enchaînements intratextuels et comme participation à un dispositif de parole inscrit dans un lieu » (Charaudeau, Maingueneau, 2002, p. 202). Contrastée à la recherche en langues anglaise et allemande, l'École française délaissait la communication professionnelle, ayant élu comme sujet de prédilection les discours à coloration idéologique ou politique. Privilégiant les théories de l'énonciation et de l'interdiscours, les linguistes de ce courant ont tardé à introduire la catégorie de genre et la seule catégorie englobante y reste toujours le « discours» (Mourlhon-Dallies, 2007).

Ces différences constituent un obstacle de plus à surmonter lors de la mise en pratique des études discursives en classe de LE. Les enseignants de différentes langues, issus de philologies diverses, utilisent des appareils conceptuels discordants et ont du mal à se comprendre en parlant des phénomènes relevant du discursif. 
Cette incompréhension résulte non seulement du fait de se référer à des auteurs et des sources qui ne sont pas connus par les partenaires de la discussion, mais aussi du fait d'attribuer des significations diverses aux termes polonais. La similitude apparente des vocables ne peut que contribuer à la confusion. L'existence des «faux-amis » terminologiques entrâne la nécessité d'établir des bases communes et de redéfinir les notions de base. L'auteure du présent article garde en souvenir plusieurs expériences de ce type, où toute discussion ultérieure devait avoir en amont une (re)définition des termes utilisés, avec le mot « discours » en tête de liste ${ }^{2}$. Aussi les études qui puisent à des sources multiples posent de sérieux problèmes terminologiques (pour la part de l'auteure du présent article, cf. Gajewska, 2013 ; Gajewska, Sowa, 2014). Par conséquent, l'introduction de l'analyse du discours en classe de LE nécessiterait non seulement un choix conscient des théories de référence à fournir aux futurs enseignants et une initiation à leur exploitation didactique, mais surtout un effort sur le plan conceptuel. Sinon, les enseignants de diverses langues étrangères continueront de se heurter aux malentendus bien connus des constructeurs de la tour de Babel et courront le risque de ne pas pouvoir mener à bien un travail en coopération ou prendre part à une conférence commune. En supposant que le discursif trouve sa place dans les classes de (diverses) LE, les apprenants qui étudient plusieurs langues seraient obligés de changer d'appareil conceptuel de référence d'un cours à l'autre, ce qui pourrait entrâner une charge cognitive supplémentaire.

\subsection{LA COMPÉTENCE DISCURSIVE SELON LE CECRL : TÉMOIGNAGE DE LA CONFUSION CONCEPTUELLE PLUS QU'UN OUTIL DE RÉFÉRENCE}

Les difficultés auxquelles on se heurte sur le plan de la définition du concept du discours et des disciplines de référence n'ont pas été surmontées en passant au concept de la compétence discursive, ce dont témoigne la liste des définitions de celle-ci dressée par Zając (2004, pp. 98-100). Hélas, le CECRL ne contribue pas à s'orienter dans ce trop-plein conceptuel et terminologique. Appariée à la compétence fonctionnelle, la compétence discursive y fait partie des compétences pragmatiques. Au pluriel au sommaire (CECRL, 2001, p. 81), au singulier par la suite, la compétence pragmatique traite de la connaissance que l'apprenant a des

\footnotetext{
${ }^{2}$ La citation qui suit illustre le problème évoqué : l'auteur utilise «dyskurs » dans une acception proche de celle en vigueur dans la terminologie allemande, c'est-à-dire pour désigner les interactions orales : «(j)eżeli 'tekstem specjalistycznym' nazwie się konkretnie graficznie lub audialnie zrealizowaną monologową wypowiedź językową, to 'dyskursem specjalistycznym' można nazwać konkretnie zrealizowany interakcyjny ciąg tekstów różnych autorów - uczestników dyskursu. Inaczej mówiąc: dyskurs specjalistyczny jest ciągiem przemiennym tekstów specjalistycznych różnych autorów, realizowanych w ramach określonej interakcji (specjalistycznej) » (Grucza, $2008: 22$ ).
} 
principes selon lesquels les messages sont organisés, structurés et adaptés (compétence discursive), utilisés pour la réalisation de fonctions communicatives (compétences fonctionnelles) et segmentés selon les schémas interactionnels et transactionnels (compétence de conception schématique, CECRL, 2001, p. 96).

La compétence discursive «permet d'ordonner les phrases en séquences afin de produire des ensembles cohérents » (CECRL, 2001, p. 96). Elle recouvre la connaissance de l'organisation des phrases et de leurs composantes et la capacité à les maîtriser en termes de thème/rhème, information donnée/information nouvelle, enchaînement «naturel » (p. ex. temporel) ou de cause/conséquence ; elle comprend aussi la capacité de "gérer et de structurer le discours en termes d'organisation thématique, de cohérence et de cohésion, d'organisation logique, de style et de registre, d'efficacité rhétorique et de principe coopératif » (maximes conversationnelles de Grice : CECRL, 2001, p. 96). La plupart des éléments listés pourraient être associés à ce qu'on qualifie parfois de «grammaire du texte». Les aspects à nette dépendance du contexte situationnel sont le registre et le style, ce premier étant toutefois cité au préalable dans le CECRL comme une des composantes de la compétence sociolinguistique où il « renvoie aux différences systématiques entre les variétés de langues utilisées dans des contextes différents » (p. 94). La compétence discursive comprend aussi «la capacité à structurer; le plan du texte»: «c'est la connaissance des conventions organisationnelles des textes dans une communauté donnée » (CECRL, 2001, p. 96), par exemple comment est structurée l'information pour réaliser les différentes macro-fonctions (description, narration, argumentation, etc.) ; comment sont racontées les histoires, les anecdotes, les plaisanteries, etc.; comment est construite une argumentation dans un débat, une cour de justice, etc. et comment les textes écrits (essais, lettres officielles, etc.) sont mis en page ou en paragraphes. La structuration du texte y est associée à une pratique sociale, par ce qu'on reconnaît le caractère institutionnalisé de la parole humaine au sein d'une communauté.

Les macro-fonctions réapparaissent par la suite dans la description de la compétence fonctionnelle. Cette dernière « recouvre l'utilisation du discours oral et des textes écrits en termes de communication à des fins fonctionnelles particulières » (p. 98). Pourtant la phrase qui suit enchaîne par la compétence conversationnelle (« (l)a compétence conversationnelle ne se réduit pas à savoir quelles formes linguistiques expriment quelles fonctions particulières (micro-fonctions)»; CECRL, 2001, p. 98). La troisième des composantes de la compétence discursive, soit la « compétence de conception schématique » n'a pas été développée, par contre les schémas d'interaction constituent une des catégories traitées dans le cadre de la compétence fonctionnelle, à côté des micro- et des macro-fonctions (CECRL, 2001, p. 98). Les dimensions du présent article ne nous permettent pas de disséquer dans le détail les composantes des compétences et les courants auxquels renvoient les diverses notions, néanmoins les exemples ici cités montrent que le CECRL ne lève pas le 
doute qui plane sur l'acception de diverses notions : au contraire, il témoigne de la confusion conceptuelle due à un embarras de richesse de courants linguistiques de référence.

\section{LES CONCEPTS DE RÉFÉRENCE : DES OUTILS À UTILITÉ RESTREINTE}

La didactique se sert de ce qui est structuré, donc analysable. Les unités, ou, de manière plus large, les modalités de description et de classement des textes proposées par les diverses approches sont nombreuses. La catégorisation des textes peut se faire à partir de la notion de genre ou de texte, on fait aussi appel (bien que nettement moins souvent) aux concepts de registre ou de style (Gajewska, 2015). Toutes ces appellations renvoient à des manières d'articuler les usages langagiers aux pratiques sociales. Or, le fait que le(s) discour(s) peu(ven)t être associé(s) au contexte communicatif de plusieurs façons et dépendre de nombreux facteurs extralinguistiques aura certainement des incidences sur leur enseignement. Dans les passages qui suivent, nous allons analyser deux cas de figure : l'exploitation des échantillons discursifs choisis par les concepteurs des manuels et leur élaboration par l'enseignant.

\subsection{LES ENSEIGNANTS FACE AUX ÉCHANTILLONS DISCURSIFS DES MANUELS : DISCERNER LES FACTEURS QUI NORMENT L'EXPRESSION}

Les termes que l'on utilise témoignent des manières de conceptualiser la langue choisies. Les études discursives font appel notamment au «genre» et au «discours », où les relations entre les deux sont complexes. La Genre Analysis anglosaxonne (cf. Bhatia, 2002, p. 17) et l'Analyse du Discours française (cf. GrzmilTylutki, 2007, p. 242) partagent la vision du genre en tant que point de croisement entre les formes linguistiques des textes empiriques et le fonctionnement social qui façonne l'expression, soit le discours. Les discours sont attachés à un certain secteur d'activité de la société à dispositif de communication particulier, impliquant des rôles, un canal ou des thèmes préférentiels (Charaudeau, Maingueneau, 2002, p. 592). Le genre apparaît alors comme un concept diadiscursif ou «transversal». L'essentiel du genre réside dans son intention pragmatique et la régularité compositionnelle qui en résulte, même s'il appartient à des sphères d'activité diverses. Ainsi donc, la lettre peut se conjuguer en lettre administrative ou pastorale et un essai académique se référer à des domaines variés (juridique, scientifique, économique...). 
Quant aux discours, leurs typologies peuvent renvoyer à deux catégories de base, nommées par Maingueneau (2005) unités transverses (auxquelles nous reviendrons en 2.2) et unités domaniales. Les unités domaniales correspondent à des espaces prédécoupés par les pratiques verbales. Maingueneau (2005) parle ici de «types de discours », attachés à un certain secteur d'activité de la société. Toutefois, la notion de type de discours apparaît comme hétérogène, car ils peuvent être groupés selon deux logiques différentes: celle de la coappartenance à un même appareil institutionnel («genres de champs » : p. ex. le discours de l'entreprise) et celle de la dépendance à l'égard d'un même positionnement (p. ex. le discours du pouvoir).

Bien que soumis à des règles d'usage plus ou moins contraignantes, tout texte (énoncé) est à la fois l'œuvre d'un émetteur unique avec ses particularités propres, qui influencent sa manière de s'exprimer. La recherche des propriétés individuelles de l'énonciateur renvoie à la stylistique et au concept de style personnel ou d'auteur. Quoique normés, les genres se prêtent de manière variée à des usages singuliers et il est désormais permis de chercher des variantes idiolectales même dans l'expression scientifique. Le style individuel peut fléchir l'usage que l'on fait du genre à un tel point que Poudat (2006, p. 33) préconise de prendre ce facteur en considération lors de la constitution d'un corpus d'analyse.

Les échantillons langagiers des manuels devraient permettre de discerner les traits qui norment l'expression. Cependant, leur nombre est d'habitude fort restreint (pour les genres du courrier électronique, cf. Gajewska, 2013, pp. 296-303) et il n'est pas rare que la déduction de ces traits doive se faire à la base d'un document unique. À partir de ce spécimen, l'enseignant (ou l'apprenant) est censé se faire une idée quant aux traits constitutifs du genre : or, il est alors quasiment impossible d'en déduire les composantes obligatoires ou constitutives. En outre, la recherche de Gajewska (2017) montre qu'il est parfois difficile d'adosser de manière univoque le type de discours aux facteurs sociaux qui le conditionnent. Par exemple, les discours en situation de travail peuvent être interprétés comme relevant du «discours d'entreprise » ou du «discours de l'entreprise Une Telle » (familiale, française ou multinationale...). De plus, les motifs de telle ou autre textualisation ne sont pas toujours évidents :

A : Bonjour, madame! Steiner de la Société ASP. Et voici Werner Bach. C'est notre ingénieur pour les systèmes d'information.

$B$ : Bonjour, messieurs! Je suis Corinne Destrade, directrice administrative. Vous venez pour la nouvelle installation? Entrez, je vous prie. Prenez place. Vous avez fait bon voyage?

A : Bonjour! Paul Smith, du cabinet Ather.

$B$ : Vous êtes consultant? Ah oui... vous travaillez à la direction des achats !

A : Tout à fait.

B : Bonjour, Paul. On s'est parlé au téléphone. Je suis Carole Vanderbek, la responsable de la communication. Je peux vous offrir un café ? Un thé? 


\section{A : Tu es stagiaire à l'informatique, non?}

B : Oui. Et toi, tu fais un stage à la direction générale. Salut, je m’appelle Éric.

A : Moi, c'est Virginie. Ça va, le travail ? (...)

(Travailler en français en entreprise ${ }^{3}$, p. 14)

Dans le guide pédagogique du manuel supracité, ses concepteurs invitent à faire réfléchir les apprenants sur le «cadre de communication ${ }^{4}$ (p. 17). Cependant, les raisons pour lesquelles Carole Vanderberk a opté, dans ses relations avec Paul Smith, pour la forme mixte « prénom + vous » ne sont pas claires, et aucune explication n'a été fournie. Finalement, il peut s'avérer problématique de discerner l'expression individuelle et la norme, même dans le cas où l'on met en scène des interactions complexes. Gajewska (2010) cite à cet égard le manuel Panorama $1^{5}$ qui ancre plusieurs dialogues didactiques dans une société fictive, Performance 2000. Les protagonistes se montrent et évoluent à travers leurs dires et leurs actions. Nous pouvons observer entre autres le directeur de l'établissement, Éric Blanc, dans ses relations avec les cadres et les employés divers, néanmoins on ne peut pas trancher s'il agit et s'il s'exprime « pour son propre compte », ou (et dans quelle mesure) en tant que représentant modèle de jeune entrepreneur, voire directeur. Lorsqu'il tutoie sa secrétaire, est-ce un marqueur qui nous montre leur degré d'intimité, le discours du pouvoir, le discours de l'entreprise (française) ou les usages de mise dans Performance 2000 ?

La limite entre règles sociales et stratégies est, dans certaines situations, difficile à cerner. Dans une conversation, il n'est pas toujours évident de repérer et de classer les éléments qui relèvent du social et ceux qui relèvent de l'individuel. Le doute aurait pu être levé par le guide pédagogique. Pourtant, afin de fournir de tels éclaircissements, les auteurs auraient dû effectuer en amont une analyse du discours respectif, ou bien au moins réfléchir sérieusement sur les intuitions langagières qui leur suggèrent une telle ou autre mise en mots des fonctions langagières présentées à travers les dialogues. Hélas, Costa (2005, p. 60) observe qu'à l'heure actuelle la conception de manuels semble reposer sur l'analyse des points forts et des points faibles des manuels existant sur le marché et sur des enquêtes de satisfaction menées auprès des utilisateurs, et que le pont entre les savoirs savants et les concepteurs de manuels devient ainsi de plus en plus invisible. Ainsi, en examinant les manuels pour l'enseignement du Français de la communication professionnelle, Gajewska (2017) arrive à la conclusion que les concepteurs des méthodes analysées semblent préférer une perspective linguistique à celle discursive : par exemple, ils présentent diverses formes adressatives, sans toutefois expliquer comment les adapter aux interlocuteurs.

\footnotetext{
${ }^{3}$ Gillmann (2007a).

${ }^{4}$ Gillmann (2007b).

${ }^{5}$ Girardet, Cridlig (2001).
} 


\subsection{L'ANALYSE DU DISCOURS AU SERVICE DE LA CONCEPTION DE MATÉRIEL PÉDAGOGIQUE}

Dans le contexte examiné ci-dessus, l'enseignant présumé était confronté à des échantillons discursifs relevant de la compétence discursive (soit de la connaissance des usages en vigueur dans une communauté discursive donnée) des concepteurs du manuel. Toutefois, il existe des contextes didactiques dans lesquels cet enseignant est censé définir lui-même les contenus du cours et fabriquer le matériel pédagogique nécessaire. Dans un «enseignement à la carte» dit «(Français) sur Objectif Spécifique » (acception de Mangiante, Parpette, 2004), l'analyse discursive va souvent de pair avec l'analyse des besoins du public cible. La mise au point d'une formation entière est un travail de taille, ainsi les méthodologies qui aident à s'en sortir avec succès sont les bienvenues. Les linguistes qui ambitionnent de trouver des solutions opérationnelles pour la collecte et l'examen des données langagières (dont Askehave, Swales, 2000 ; Bhatia, 1993 ; Mourlhon-Dallies, 20086) optent pour le genre en tant qu'unité de référence. Cette unité s'est avérée aisée à manier (soit à analyser et à didactiser) du fait de sa dimension restreinte, car conditionnée par la situation de communication respective, au sein de laquelle un genre se stabilise. Hélas, le fait que le genre est issu d'une action langagière récurrente détermine en même temps les limites de son application. La didactique des langues tire profit essentiellement des genres routiniers, notamment des genres appelés par Maingueneau (2004) «genres institués de mode (1)», lesquels se stabilisent par des pratiques communicatives répétitives. Or, une grande partie des interactions orales et électroniquement médiées (en ce qui concerne ces dernières, cf. Gajewska, 2013, pp. 255-268) sont peu formatées et pour cette raison difficiles à décrire et à enseigner.

Le genre n'est pas la seule «unité transverse » (représentation reprise à Maingueneau, 2005) qui traverse les textes relevant de multiples types de discours. Outre les genres, les linguistes voient d'autres «modes de structuration fondamentaux » (Charaudeau, Maingueneau, 2002, p. 592) ou «formes d'organisation linguistique » (Bronckart 1996, p. 254) qui se combinent dans les textes effectifs. Ces unités peuvent être définies à partir de critères linguistiques, fonctionnels ou communicationnels. Ces derniers combinent traits linguistiques, fonctionnels et sociaux : nous pouvons classer ici les diverses propositions de découpage de la communication spécialisée en paliers, allant du discours de la vulgarisation au discours de la recherche (entre autres celles de Freddi, 1988 ; Cabré, 1993, Widdowson, 1979). Parmi les typologies qui opèrent au niveau des formes d'organisation textuelle, les plus connues sont celles établies par Charaudeau (1983 : ordre énonciatif, argumentatif, narratif et rhétorique), Adam (1990 : séquence narrative, descriptive, argumentative,

\footnotetext{
${ }^{6}$ Dans une perspective axée davantage sur l'enseignement sur objectifs généraux, aussi Béacco (2007) met au centre de l'approche par compétences le genre de discours.
} 
explicative, argumentative, expositive-explicative, injonctive-instructionnelle, conversationnelle et poétique-autotélique) et Bronckart (1996 : séquence narrative, descriptive, argumentative, explicative, dialogale).

Cependant, à notre savoir ces formes d'organisation textuelle n'ont pas été pourvues de méthodologies qui les rendraient applicables à des contextes discursifs particuliers, notamment si nous envisageons qu'elles seraient utilisées non par des chercheurs en sciences du langage, mais par des didacticiens du terrain. Contrairement aux genres, ces catégories ne sont pas intuitives pour les utilisateurs (notons que la plupart des genres usuels portent des noms donnés par leurs usagers). Les séquences bronckartiennes sont forcément présentes dans les manuels examinés par Zając (2004), sans toutefois être expliquées en tant que telles et à l'aide des concepts proposés par le linguiste. D'ailleurs Zając aborde les documents retenus par le biais des genres discursifs (catégorie qu'elle explicite dans les en-têtes de ses grilles d'analyse) : ainsi, le tableau récapitulatif rassemblant les séquences explicatives/ démonstratives dénombre reportages, interviews, lettres officielles, courrier électronique, affiches etc., ce qui témoigne que les séquences de Bronckart ne semblent pas opérationnelles en elles-mêmes et demandent un complément pour que les textes évoqués puissent être référencés de manière univoque. Ce trait met en doute leur utilité en tant que concept de base pour l'élaboration des cursus ou des démarches didactiques efficaces ${ }^{7}$.

\section{UN OBJET D'ÉTUDE HYBRIDE ET INSTABLE}

Le recours à l'analyse du discours s'avère compliqué non seulement dans les cas où la communication est peu formatée et les unités d'analyse qui sont censées remplacer le genre ne se montrent pas aussi opérationnelles: les usages discursifs rendent dure la tâche des chercheurs par leur nature instable et changeante. Chaque sphère d'activité humaine génère des types d'expression propres, qui peuvent par la suite se stabiliser en un répertoire de catégories normatives, soit de genres. L'ensemble des genres disponibles (genre network de Swales, 2004) reflète les relations entre les acteurs et la logique de procéder et de communiquer dans une communauté discursive donnée. Le répertoire des types reste stable jusqu'au moment où cette communauté n'identifie une situation pour nouvelle et exigeant par conséquence une réponse rhétorique innovante. Ceci assure au même temps l'évolution des genres et des discours et leur adaptation aux changements sociaux. Les genres peuvent changer également grâce à la créativité personnelle : les locuteurs experts

\footnotetext{
${ }^{7}$ Pour une analyse plus ample des limites d'une approche par les séquences textuelles, voir Richer (2011).
} 
sont capables de «fléchir» un genre donné afin d'exprimer des intentions propres ou d'atteindre des objectifs particuliers. La naissance d'un genre se fait à la croisée du collectif et de l'individuel : «half someone else's/half our own» (Norris, Jones, 2005, p. 7). Par la répétition des actions individuelles, le collectif se sédimente; à travers l'agir individuel, les pratiques sociales muent.

Souvent, un genre évolue à partir de types existants. Fairclough (1992) introduit ici le concept d'interdiscursivité (interdiscursivity), notion importante lors de l'analyse des genres d'expression anglaise. Les frontières entre un discours et l'autre sont en effet confuses, car un texte peut relever de plusieurs discours. Entre autres, le contact des textes et des discours générés dans le cadre des multinationales constitue ici un facteur de taille. Les discours spécialisés sont alors sujets aux pressions de la variation interculturelle, car ce n'est pas seulement les facteurs socio-culturels inhérents à un texte, mais aussi les schémas d'interprétation qui affectent profondément sa réalisation au sein de la communauté professionnelle qui l'adopte (Gotti, 2003).

Bien que les chercheurs aient parfois tendance à identifier des objets textuels en termes de «genres purs », dans la pratique on a souvent affaire à des formes mixtes, soit parce qu'ils poursuivent plusieurs objectifs pragmatiques à la fois, soit du fait de viser des objectifs propres dans le cadre d'une autre forme générique à objectifs assignés. Dans un monde en évolution, de nombreux genres s'ouvrent à de nouveaux buts communicatifs occasionnant un «mélange de genres » (genre mixing: Bhatia, 1997). Bhatia cite dans ce contexte entre autres l'intrusion croissante des éléments promotionnels dans des genres d'information aussi divers que des éditoriaux de presse, documents juridiques et rapports. L'appropriation des ressources d'un genre afin de les utiliser pour la construction d'un autre, soit les cas dans lesquels une forme générique particulière est utilisée comme modèle pour donner expression à une autre forme générique conventionnelle distincte, donnent naissance à des « genres enchâssés » (genre embedding : Bhatia, 1997). Ce procédé est très fréquent en publicité, où par exemple une annonce peut être rédigée dans le format d'un poème. Les genres qui présentent à la fois ces deux propriétés («both mixed and embedded») sont considérés par Bhatia (2004, p. 90) comme des «genres hybrides » (hybrid genres).

La constatation que les genres sont en évolution constante n'allège certes la tâche du chercheur ni de l'enseignant qui voudrait fournir à ses apprenants des recettes discursives toutes faites, dans l'espoir qu'elles leur suffisent pendant des années. Par contre, elle encourage ceux qui voudraient participer de manière active à l'élaboration de normes sociolinguistiques. L'Analyse Critique du Discours (Critical Discourse Analysis) préconise d'abandonner l'objectivité scientifique envers l'objet de l'étude pour une attitude critique afin d'améliorer les pratiques sociales, par exemple éliminer les comportements langagiers discriminatoires. 


\section{EN GUISE DE CONCLUSION : FORMER À DÉVELOPPER DE MANIÈRE AUTONOME SA COMPÉTENCE DISCURSIVE}

Si enseigner une langue, c'est enseigner à communiquer, l'objectif majeur de l'enseignement est d'amener l'apprenant à communiquer dans la langue étrangère. Afin que ses interventions soient acceptées par les interlocuteurs, il doit tenir compte des normes sociales qui pèsent sur l'activité verbale dans une situation déterminée et intégrer les règles discursives en tant que données qui influencent la communication. Les fondements ou les modèles théoriques des règles d'usage sous-jacentes à la communication sont de toute première importance dans l'enseignement des langues étrangères. De telles connaissances, utiles lors de l'examen des échantillons langagiers contenus dans les manuels de (F)LE afin de discerner ce qui est accessoire et individuel de ce qui est socialement marqué, deviennent indispensables lorsque l'enseignant est obligé de concevoir par lui-même des contenus et des matériels didactiques, cas courant dans l'enseignement dit «(Français) sur Objectif Spécifique ».

Or, pour l'instant, un hiatus semble exister entre la théorie et la pratique didactique. D'un côté, nous constatons une multitude de courants qui analysent l'expression langagière ancrée dans le contexte. Pourtant, dès que nous nous tournons du côté de la pratique didactique, comme dans les recherches citées dans le présent texte (Gajewska, 2010, 2013, 2017), nous constatons que cette pratique reste étrangement étanche aux avancées de la recherche en AD. La divergence des cadres conceptuels potentiels peut être un des freins qui stoppent le progrès dans ce domaine. Une abondance des modèles de référence n'est pas un obstacle en soi, pourtant elle peut décourager les publics impliqués : les concepteurs des manuels, les enseignants et finalement les apprenants. Néanmoins, il est vital d'encourager les concepteurs des manuels à mettre l'AD en pratique lors du rassemblement des échantillons langagiers et à en fournir des analyses plus fines; d'inciter les enseignants à faire décrypter les liens entre les facteurs contextuels et la mise en texte, de former les apprenants à regarder la communication sous cet angle et à mettre ces connaissances à leur profit pendant la communication exolingue.

L'espoir de dépasser enfin cet obstacle pourrait venir des études qui envisagent le discours dans une perspective actionnelle. La didactique et la linguistique voient actuellement la communication dans son ensemble, aspects non verbaux y compris. Sur ce terrain, elles peuvent assez facilement trouver des fondements communs et élaborer des approches conjointes. L'Analyse des Discours Médiée (Mediated Discourse Analysis) de Scollon (2001) préfère à la notion de genres du discours le « maillage de pratiques » (nexus of practice). Ce concept, repris en linguistique entre autres par des linguistes du groupe de recherche genevois «Langage, Action, Formation » et en didactique par Mourlhon-Dallies (2008) dans son Analyse Différentielle des Discours, a une visée résolument pratique et s'accorde bien avec les postu- 
lats de l'approche actionnelle. Appartenant à la fois au collectif et à l'individuel, il inclut à la fois le non-verbal, le dit et les non-dits (le savoir préalable) qui structurent les activités routinières. C'est de ce côté-là que l'on pourrait donc creuser afin d'élaborer un modèle d'analyse et de description des normes discursives, qui pourrait par la suite orienter la réflexion sur la compétence discursive, ses composantes et ses échelles de progression.

La didactique - et pas seulement la didactique des LE - est orientée sur l'acquisition de savoir et savoir-faire concrets, comme les normes discursives et des manières de faire dans une situation donnée (soit les genres). La formation professionnelle inclut entre autres l'apprentissage des normes communicatives propres à la communauté professionnelle, à laquelle l'apprenti aspire : Devitt (1991, p. 140) constate même qu'un «système de genres » accomplit le travail dans les situations de travail récurrentes. Le texte présent est orienté davantage sur l'étude et la didactisation de ce qui est social dans l'expression verbale. Pourtant, comme il a été signalé, les normes discursives sont souvent en mutation et les genres peuvent emprunter - ou prêter - des traits formels à d'autres genres. Ainsi, l'enseignement des LE dans une perspective discursive devrait prévoir non seulement une sensibilisation à l'existence des usages langagiers dépendant des facteurs sociaux, mais aussi un éveil aux stratégies qui permettraient de les détourner en sa faveur, d'être un utilisateur critique et conscient des normes en vigueur dans les sphères d'activité dans lesquelles on opère, au lieu de s'y plier aveuglément. Dans une telle acception, le discours (Wilczyńska, 2013) et la compétence discursive apparaissent comme un élément incontournable, et même une clé de voûte, dans la formation langagière et personnelle. 


\section{BIBLIOGRAPHIE}

Adam, J.-M. (1990). Éléments de linguistique textuelle, théorie et pratique de l'analyse textuelle. Madraga : Liège.

Askehave, I., Swales, J. M. (2000). Genre identification and communicative purpose: A problem and possible solution. Applied Linguistics, 22, 195-212.

Béacco, J. C. (2007). L'approche par compétences dans l'enseignement des langues. Paris : Didier.

Bérard, E. (1991). L'approche communicative, théories et pratiques. Paris : CLE International.

Bhatia, V. K. (1993). Analysing Genre. Language Use in Professional Settings. London - New York: Longman.

Bhatia, V. K. (1997). Genre-mixing in academic introductions. English for Specific Purposes, 16(3), 181-195.

Bhatia, V. K. (2002). Applied genre analysis: a multi-perspective model. Ibérica, 4, 3-19. http://www. aelfe.org/documents/text4-Bhatia.pdf

Bhatia, V. K. (2004). Words of Written Discourse : A Genre-Based Viev. London : Continuum.

Boutet, J., Maingueneau, D. (2005). Sociolinguistique et analyse de discours : façons de dire, facons de faire. Langage et société, 114, 15-48.

Bronckart, J. P. (1996). Activité langagière, textes et discours. Pour un interactionnisme social. Lausanne - Paris : Delachaux et Niestlé.

Cabré, M. T. (1993). La terminología. Barcelona : Antártida.

Candlin, C. N. (1993). General Editor's Preface. In V. K. Bhatia (réd.), Analysing Genre. Language Use in Professional Settings (pp. IX-XI). London : Longman.

Charaudeau, P. (1983). Langage et discours. Paris : Hachette.

Charaudeau, P., Maingueneau, D. (réd.). (2002). Dictionnaire d'analyse du discours. Paris : Le Seuil.

Chomsky, N. (1965). Aspects of the Theory of Syntax. Cambridge : M.I.T. Press.

Conseil de l'Europe (2001). Cadre Européen Commun de Référence pour les Langues: Apprendre, Enseigner, Évaluer. Strasbourg : Didier/Conseil de l'Europe.

Costa, P. (2005). Compétence de communication et didactique des langues étrangères : la liaison ratée !, Synergies Pologne, 1, 56-61. URL : https://gerflint.fr/Base/Pologne1/competence.pdf

Czachur, W., Miller, D. (2002). Niemiecka lingwistyka dyskursu - próba bilansu i perspektywy. Oblicza komunikacji, 5, 25-43.

Devitt, A. J. (1991). Intertextuality in tax acconting generic, referential and functional. In Ch. Bazerman, J. Paradis (réd.), Textual Dynamics of the Professions: Historical and Contemporary Studies of Writing in Professional Communities (pp. 336-357). Madison, WI : University of Wisconsin Press.

Fairclough, N. (1992). Discourse and Social Change. Cambridge : Polity.

Freddi, G. (1988). Linee per una didattica delle microlingue. In CLUC, Il linguaggio delle scienze e il suo insegnamento. Brescia : La Scuola.

Gajewska, E. (2010). Przygotowanie do pracy w przedsiębiorstwie międzynarodowym na lekcji JO. In M. Mackiewicz (réd.), Kompetencja interkulturowa w teorii i praktyce edukacyjnej (pp. 221-230). Poznań : Wyd. WSB.

Gajewska, E. (2013). Courriel versus courrier : la communication écrite en français de la communication professionnelle au temps des nouvelles technologies. Lublin : Werset.

Gajewska, E. (2015). Badania nad dyskursami pedagogicznymi a praktyka pedagogiczna. In M. Sowa, M. Mocarz-Kleindienst, U. Czyżewska (réd.), Nauczanie języków obcych na potrzeby rynku pracy (pp. 39-52). Lublin : Wyd. KUL.

Gajewska, E. (2017). Analiza dyskursu w nauczaniu języków obcych do celów zawodowych na przykładzie 'Français de la communication professionnelle'. Lingwistyka Stosowana, 22, 15-29. 
Gajewska, E., Sowa, M. (2014). LSP, FOS, Fachsprache... Dydaktyka języków specjalistycznych. Werset : Lublin.

Gillmann, B. (2007a). Travailler en français en entreprise. Paris : Didier.

Gillmann, B. (2007b). Travailler en français en entreprise. Guide pédagogique. Paris : Didier.

Girardet, J., Cridlig, J.-M. (2001). Panorama 1, Paris : CLE International.

Gotti, M. (2003). Specialized Discourse. Linguistic Features and Changing Conventions. Bern : Peter Lang.

Grzmil-Tylutki, H. (2007). Gatunek w świetle francuskiej teorii dyskursu. Kraków : Universitas.

Grucza, S. (2008). Lingwistyka języków specjalistycznych. Warszawa : Katedra Języków Specjalistycznych i Katedra Teorii Języków i Akwizycji Językowej UW/Euro-Edukacja.

Hymes, D. H. (1966). Two types of linguistic relativity. In W. Bright (réd.), Sociolinguistics (pp. 114-158). The Hague : Mouton.

Hymes, D. H. (1984). Vers la compétence de communication. Paris : Hatier-Crédif.

Maingueneau, D. (2004). Typologie des genres de discours institués. URL : http://dominique.maingueneau. pagesperso-orange.fr/intro_topic.html

Maingueneau, D. (2005). L'analyse du discours et ses frontières. Marges Linguistiques, 9, 64-75. URL: http://www.revue-texto.net/Parutions/Marges/00_ml092005.pdf

Maingueneau, D. (2014). Discours et analyse du discours. Introduction. Paris : Armand Colin.

Mangiante, J. M., Parpette, C. (2004). Le Français sur Objectif Spécifique : de l'analyse des besoins à l'élaboration d'un cours. Paris : Hachette.

Mourlhon-Dallies, F. (2007). Communication électronique et genres du discours. Glottopol, 10. URL: http://www.univ-rouen.fr/dyalang/glottopol/telecharger/numero_10/gpl10_01mourlhon

Mourlhon-Dallies, F. (2008). Enseigner une langue à des fins professionnelles. Paris : Didier.

Norris, S., Jones, R. H. (réd.). (2005). Discourse in Action: Introducing mediates discourse analysis. London - New York : Routledge.

Poudat, C. (2006). Étude contrastive de l'article scientifique de revue linguistique dans une perspective d'analyse des genres. Texto!, 11(3-4). URL: http://www.revue-texto.net/1996-2007/Corpus/ Corpus.html

Richer, J. J. (2011). Les genres de discours : une autre approche possible de la sélection de contenus grammaticaux pour l'enseignement/apprentissage du F.L.E. ?. Linx, 64-65, 15-25. URL : http://linx. revues.org/1396

Sanders, C. (1996). Regards anglo-saxons sur les registres et les genres discursifs. Le Français dans le Monde. Recherches et Applications «Le discours : enjeux et perspectives », 46-54.

Scollon, R. (2001). Mediated discourse: The nexus of practice. London - New York : Routledge.

Swales, J. M. (2004). Research Genres. Exploration and Application. Cambridge : Cambridge University Press.

Widdowson, H. G. (1979). Explorations in Applied Linguistics. Oxford : Oxford University Press.

Wilczyńska, W. (2013). Dyskurs - brakujące ogniwo nierozerwalności kultury i języka, czyli o integracji w kształceniu neofilologów. Lingwistyka stosowana, 8, 133-152. URL : http://www.ls.uw.edu. pl/documents/7276721/11196137/LS8_2013_art_Wilczynska.pdf

Zając, J. (2004). Du communicatif au discursif. Approches de la pragmatique du discours à la didactique des langues étrangères. Warszawa : Instytut Romanistyki, Uniwersytet Warszawski. 